\title{
Agôn
}

Revue des arts de la scène

HS 2 | 2014

Mettre en scène le conte

\section{Les métamorphoses scéniques de Cendrillon : de la féérie à l'inquiétante étrangeté}

\section{Rafaëlle Jolivet Pignon}

\section{(2) OpenEdition}

\section{Journals}

Édition électronique

URL : http://journals.openedition.org/agon/3158

DOI : $10.4000 /$ agon.3158

ISSN : 1961-8581

Éditeur

Association Agôn

Référence électronique

Rafaëlle Jolivet Pignon, « Les métamorphoses scéniques de Cendrillon : de la féérie à l'inquiétante étrangeté », Agôn [En ligne], HS 2 | 2014, mis en ligne le 09 mars 2015, consulté le 01 mai 2019. URL : http://journals.openedition.org/agon/3158; DOI : 10.4000/agon.3158

Ce document a été généré automatiquement le 1 mai 2019.

Association Agôn et les auteurs des articles 


\title{
Les métamorphoses scéniques de Cendrillon : de la féérie à l'inquiétante étrangeté
}

\author{
Rafaëlle Jolivet Pignon
}

\section{Cendrillon, une figure protéiforme et sans âge}

1 L'origine orale et les multiples variations de l'histoire de Cendrillon lui ouvrent l'horizon de tous les possibles devenirs scéniques. On a souligné ${ }^{1}$ comment la simplicité du récit de Perrault se prête particulièrement à une adaptation pour la scène. Sans âge - on trouve des récits s'apparentant au canevas type de ce conte à partir du $1^{\text {er }}$ siècle avant Jésus$\mathrm{Christ}^{2}$ - , la fable se plie à des variations innombrables parce qu'elle expose un parcours initiatique vers la réalisation de soi, une sortie de chrysalide vers la lumière. Rappelons rapidement les éléments récurrents dans les différentes versions : le passage du malheur au bonheur d'une jeune fille orpheline et maltraitée, l'intervention du merveilleux, le soulier perdu, identification de sa propriétaire, et son union avec le prince. Cette simplicité, presque trop lisse pourrait-on ajouter, est un appel à la variation mais aussi à l'adaptation et à la transposition: chaque auteur (qu'il soit écrivain, musicien, chorégraphe, cinéaste, etc.) en propose sa propre lecture, liée au goût de l'époque, s'écartant parfois de manière très nette du modèle d'origine.

2 Le conte de Perrault est ainsi adapté pour la scène dès 1759 avec l'opéra comique écrit par Louis Anseaume, et sa postérité adopte les formes les plus variées : théâtre, mais aussi pantomime, vaudeville, mélodrame, opérette, opéra, opéra-ballet, ballet, marionnettes, sans parler du cinéma (de Méliès en 1906 à Kenneth Branagh en 2015 en passant le fameux dessin animé de Walt Disney en 1950). Cette faculté d'adaptation met en lumière la plasticité du conte, et montre à quel point il a su capter l'intérêt des artistes dans tous les domaines et à toutes les époques jusqu'à aujourd'hui. Quel qu'en soit sa forme artistique et l'époque dans laquelle il s'inscrit, Cendrillon est chaque fois, par la relecture que le spectacle propose, un miroir tendu, un questionnement porté sur ses 
contemporains : qu'est-ce que cette histoire nous dit de nous-mêmes et de notre monde ? Par ailleurs, parce qu'il pose des problèmes à la scène (comment montrer le merveilleux ? ), l'adaptation de Cendrillon inclut de facto une dimension métathéâtrale sur sa fabrique scénique et se positionne clairement sur son rapport au réel: faut-il encore croire au merveilleux ou n'est-ce qu'un simple trucage de théâtre?

Dans ce foisonnement des formes et des propositions artistiques, nous nous interrogerons sur l'invention de la forme scénique en relation avec le merveilleux, nous montrerons comment la dramaturgie scénique du conte joue sur le déplacement des genres artistiques et nous envisagerons comment la modernité prend à rebours l'idée de merveilleux pour lui substituer la notion d'« inquiétante étrangeté ».

\section{Les pouvoirs scéniques de la féérie}

Dès la fin du xvIII ${ }^{e}$ siècle, le goût pour le merveilleux donne lieu à de nombreux spectacles jouant sur les trucages et astuces de mise en scène, tandis que la machinerie théâtrale s'ingénie aux effets d'escamotage ou d'apparitions. Les changements à vue qui consistent à changer le décor sans baisser le rideau sont très appréciés. Le genre de la féérie ${ }^{3}$, qui stimule particulièrement l'invention scénique, est alors en plein essor et verra sa consécration au XIX ${ }^{\mathrm{e}}$ pour un public toujours plus avide d'innovations visuelles. La fin des années 1830 voit ainsi émerger la grande féerie à tableaux, associant burlesque, vaudeville et changements à vue spectaculaires. Le genre s'épanouit dans les décennies qui suivent, multipliant tous les effets visuels liés aux avancées de la machinerie au théâtre et en optique si bien que mise en scène et technologie semblent intimement liées. Ces spectacles sont cependant si coûteux qu'ils se raréfient après la guerre de 1870 : on ne compte plus qu'une création par saison, cantonnée au Châtelet.

\section{Les possibilités technologiques au service de l'illusion}

5 L'avènement de la figure du metteur en scène, à la fin du XIXe siècle, est contemporain d'avancées techniques et notamment de l'arrivée de l'éclairage à l'électricité permettant une recherche esthétique tout en élargissant les possibilités de jeu et de mise en scène. L'Opéra Comique où est créé Cendrillon, opéra de Jules Massenet sur le livret d'Henri Cain en 1899, se place en effet sous l'égide de la fée électricité, nouvel éclairage de la salle Favart qui remplace le système de l'éclairage à gaz. Cette technologie permet tout un jeu sur les couleurs et des changements d'intensité en relation avec la palette orchestrale et vocale. Comme le souligne le metteur en scène Albert Carré : "La lumière devient un moyen d'expression d'une infinie souplesse, qui renforce l'atmosphère du drame, anime le décor ou le suggère, et souligne la présence dramatique de l'acteur »4. Le Menestrel, journal qui avait salué cette innovation technique écrivit à propos de cette création : « Des groupes de nymphes font voler leurs écharpes et dansent silencieusement autour du chêne, sous le ciel bleu, poursuivant leurs évolutions harmonieuses jusqu'au bord du lac dont les ondes transparentes brillent là-bas, sous la mate clarté de la lune $»^{5}$. L'enchantement opère, le public s'extasie devant cette Fée Électricité qui se confond alors avec la marraine de Cendrillon. 


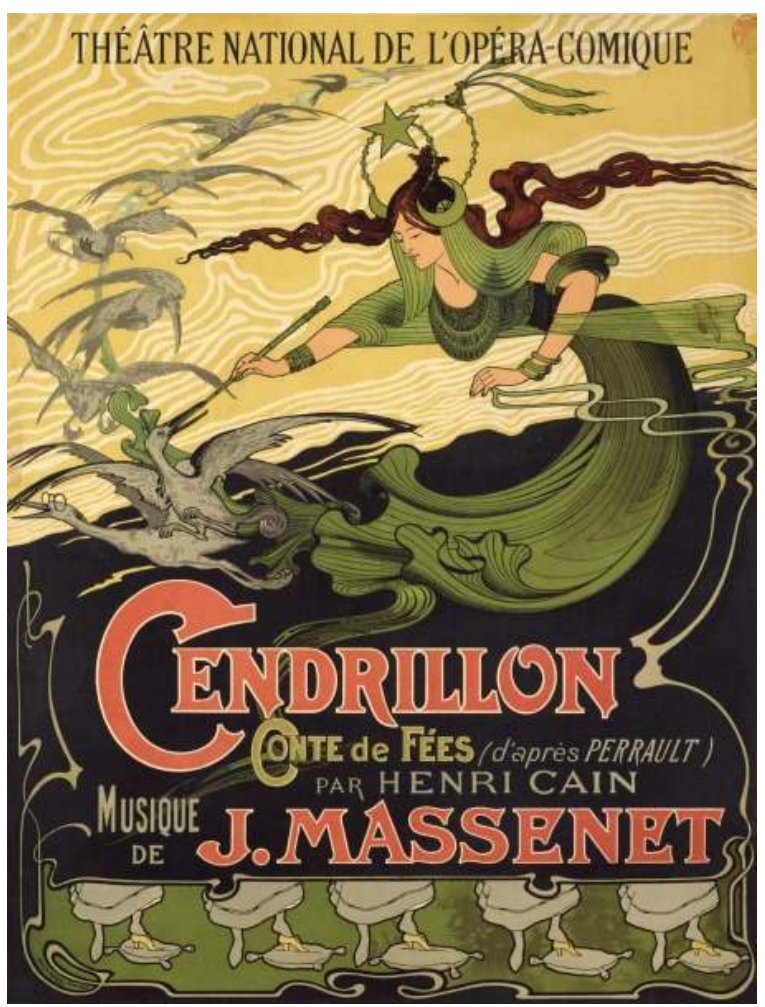

Bibliothèque nationale de France, Bibliothèque-musée de l'Opéra (Aff 649)

André Devambez (c) Adagp, Paris 2001

Le personnage principal dans cette version scénique du conte est bien la Fée, comme en témoigne l'affiche du spectacle de la création, et non Cendrillon.

\section{Les « clous » du spectacle}

Dans nombre Fééries, le conte n'est souvent qu'un prétexte qui permet un déploiement de virtuosités scéniques dont le public raffole; c'est ainsi que la multiplicité des tableaux proposent un voyage de «clou » en «clou» dont la machinerie est le ressort principal. Une caricature de Gill dans La Lune représente ainsi une modeste chaussure légendée : "Ci-jointe la pantoufle du seul artiste de la maison, M. le machiniste ». Dans l'opéra de Massenet cependant, la mise en scène de Carré est au service de l'œuvre musicale qui ne cède en rien aux effets spéciaux, même s'ils ponctuent le livret et sont effectivement très attendus. Le metteur en scène recourt à trois trucages successifs : la transformation de Cendrillon par sa marraine la Fée, l'apparition du carrosse enchanté et enfin, la branche articulée du Chêne des Fées. Chacun de ces trucages, décrits par Carré dans son livret de mise en scène, résulte en fait d'une injonction magique de la Fée, qui rend alors indispensable leur convocation: "Cendrillon tu seras la beauté sans pareille!», "Voici ton carrosse, princesse! » et «Fermez-vous muraille embaumée!». Les effets associés à ces paroles sont si bien réalisés que la magie opère sur le spectateur ébloui. Comme le stipulait le prologue qui a finalement été supprimé, le spectacle a pour visée principale de faire rêver le public et pour cela il cherche à créer une illusion parfaite. La mise en scène 
est alors un moyen au service de cette fête des sens et le spectateur est invité à oublier le réel pour entrer dans l'univers du merveilleux.

\section{Relectures dramaturgiques et mélange des genres scéniques}

\section{Une joyeuse hybridation des registres et des formes}

Peu de textes auront donné une telle postérité à leur modèle. Cendrillon de Perrault est non seulement infiniment repris, réécrit, voire détourné sur le plan dramaturgique (les modifications touchent tous les éléments de l'histoire), mais il connaît de plus de multiples adaptations selon les genres en vogue et selon la spécificité du théâtre qui le produit ${ }^{7}$. Parallèlement à la rêverie et au merveilleux, une veine comique, grivoise et parodique fait les délices d'un large public. Il est frappant de constater que la multiplication des Cendrillon construit de version en version une connivence avec le spectateur averti : "Tant de gens ont essayé cette pantoufle, qu'il n'est pas étonnant qu'elle soit élargie » entend-on dans La Famille des Cendrillon. Cendrillon passe d'une forme théâtrale à une autre (opéra, vaudeville, mélodrame, ballet) en changeant de théâtre et s'épanouit dans des formes hybrides associant danses et chants. Les ajouts musicaux dans les vaudevilles sont souvent des airs connus du public qui s'amuse de ces effets de reconnaissance et reprend en chœur avec les chanteurs. Ce joyeux choc des registres que la partition soit parlée ou chantée nourrit la variété des inventions : du pathétique au comique, du tragique au burlesque, du parodique au grivois. Citons parmi les créations les plus marquantes et les plus représentées la Cendrillon de Louis Anseaume (1759), cousine des contes licencieux du XVIII ${ }^{\mathrm{e}}$ siècle, qui commence au lendemain du bal où la jeune fille n'ose avouer à sa marraine ce qu'elle a perdu dans la nuit. Le triomphe du badinage libertin s'achève sur l'essai de la pantoufle par le prince lui-même, au milieu de la cour. Après la Révolution cependant, la scène parisienne n'apprécie plus beaucoup l'érotisme et se méfie des fées, le livret de Charles-Guillaume Etienne écrit pour le compositeur maltais Nicolas Isouard, dit Nicolò, insiste sur le côté touchant de l'héroïne. Le prince, qui échange ses habits avec son écuyer pour éprouver les véritables sentiments des sœurs, est à l'école des Lumières lorsqu'il suit les conseils du précepteur un peu sentencieux qui remplace la fée. Le larmoyant n'est pas loin dans cet opéra-féérique (qui a relativement évacué le merveilleux) créé le 24 mai 1810 à l'Opéra-Comique. Le succès est phénoménal durant toute la période impériale où il est donné plus d'une centaine de fois et inspire l'année même de sa création six parodies. Traduit en italien, le livret de La Cenerentola de Rossini (1817), à peine démarqué de celui d'Etienne, présente la même histoire, sans fée ni pantoufle, mais avec les mêmes personnages portant les mêmes noms. Très vite dans toute l'Europe, le public succombe au charme de la partition de Nicolò que l'OpéraComique reprend en 1845 dans une nouvelle orchestration d'Adolphe Adam, puis en 1877 avec ajout de danses et rétablissement de la fée! Parmi les spectacles remarqués, citons aussi la grande féerie de Clairville, d'Albert Monnier et d'Ernest Blum, créée en 1866, au Châtelet, et dont le succès (le spectacle est repris jusqu'en 1888) fut proportionnel à sa démesure: avec vingt-six acteurs-chanteurs, cent danseuses pour trois heures de spectacle, cette Cendrillon cherche à en faire toujours plus. Enfin, c'est en 1899 qu'est créé à l'Opéra-Comique l'œuvre lyrique de Massenet sur le livret d'Henri Cain. Cette version 
propose une féerie poétique et légère sans dramatisme réaliste dans laquelle les personnages gardent la simplicité profonde du conte.

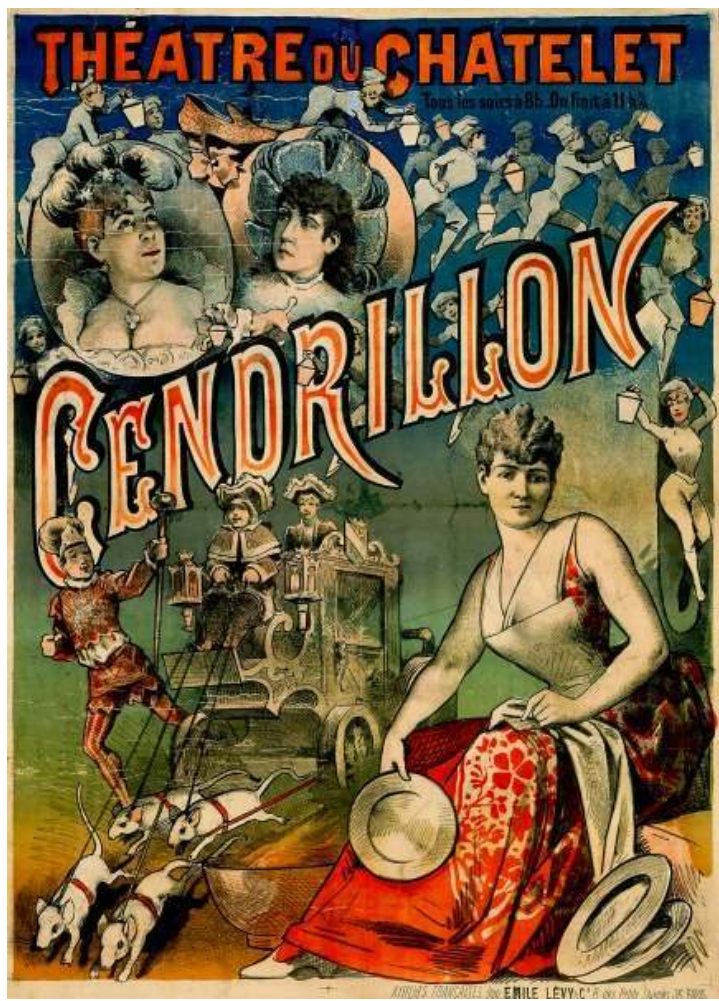

Bibliothèque nationale de France, Estampes et Photographie (LEVY Émile, Rouleau n³)

(c) Bibliothèque nationale de France

\section{Cendrillon et le ballet : la vision hollywoodienne de Rudolf Noureev}

Le personnage de Cendrillon a peu retenu l'attention des chorégraphes avant 1945 et la composition musicale de Sergei Prokofiev ${ }^{8}$. C'est à travers cette musique qu'il devient une des figures importantes du patrimoine du ballet. La Cendrillon de Prokofiev est conforme au conte, préservant les éléments de l'histoire, mais elle s'en éloigne par son ampleur, notamment son abondance de personnages et de situations qui noient passablement l'intrigue première. Aussi la transformation de Cendrillon fait-elle intervenir les Fées des Quatre Saisons, le bal présente une série de danses de courtisans, et, dans sa recherche du pied pouvant chausser la pantoufle, le prince parcourt les cinq continents (d'où les diverses danses exotiques). Nombreux chorégraphes contemporains s'appuient sur cette partition musicale pour construire leur chorégraphie : Maguy Marin à l'opéra de Lyon (1885), Rudolf Noureev à l'opéra de Paris (1986), Jean-Christophe Millot et les Ballets de Monte-Carlo (1999), Thierry Malandain et le ballet de Biarritz (2013) entre autres.

Avec la complicité de son décorateur Petrika Ionesco, Rudolf Noureev transpose l'histoire de Cendrillon dans l'univers hollywoodien des années 30 comme il l'explique dans sa note d'intention :

Lorsque Petrika Ionesco m'a soufflé l'idée d'une Cendrillon hollywoodienne, j'ai commencé par être très réticent : je craignais une déformation abusive du conte de 
Perrault. Dois-je regretter que cette suggestion se soit insidieusement glissée dans ma tête, au point de ne plus me lâcher ?J'ai finalement dit oui, et aussitôt j'ai travaillé à la chorégraphie, en fonction de cette idée. L'époque est celle des années 30 et 40 . Celle d'un moment de la vie de Prokofiev, où rentré dans son pays, l'URSS, il éprouvait pour l'Occident une sourde nostalgie. Cendrillon n'est pas très russe. C'est même ce qu'il a fait de plus occidental. Non seulement la musique dicte le ton, mais les danses sont décalées par rapport au contexte. C'est ce décalage que nous avons voulu rendre, en transposant le conte dans l'univers du cinéma. Cependant, dans cette version, la mécanique de l'histoire n'a pas changé. On y retrouve les deux sœurs ridicules et diaboliques, la terrible marâtre, le père tiraillé entre sa nouvelle femme et cette jeune fille - Cendrillon - sa fille, dont il sait bien qu'elle est maltraitée et doit, pour survivre, se réfugier dans le rêve d'une vie qui lui est interdite. Tout le drame de Cendrillon, c'est la marche du temps, la peur de voir son rêve s'écrouler, son bonheur fuir avec sa jeunesse. C'est pour cela qu'elle se sauve au moment où l'amour la transfigure. Moi-même, je conçois la vie éternelle comme un luxe suprême! Le ballet Cendrillon est devenu un rêve de cinéma. Un rêve de robe blanche, teintée d'un peu de rose pour rendre hommage à l'innocence, légèrement argentée aussi, parce que Cendrillon est un personnage d'aujourd'hui, elle ne rêve qu'à une chose : devenir star. Dans ma version de Cendrillon, la fée s'est alors métamorphosée en producteur de cinéma, seul personnage de la mythologie moderne capable, par la magie de son art, de transformer une citrouille en carrosserie de voiture? .

11 Découverte par un producteur de cinéma (interprété par Rudolf Noureev lui-même), la jeune fille modeste (Sylvie Guillem) échappe à un père alcoolique et à une marâtre odieuse pour faire ses débuts à l'écran, accrochant au passage le cœur de l'acteur principal. Dans cette transposition artistique, Noureev cherche la féerie contemporaine : le cinéma américain et les studios d'Hollywood semblent des plus appropriés aujourd'hui pour faire rêver les jeunes filles. Les deux sœurs de Cendrillon fréquentent les producteurs dans l'espoir d'être repérées... La marraine est devenue un producteur, les Fées des Quatre saisons sont remplacées par un défilé de mode; le palais du prince devient le studio de tournage de King-Kong, la recherche du prince à travers les cinq continents fait place à une tournée des boîtes de nuit exotiques. 


\section{Cendrillon dansant}

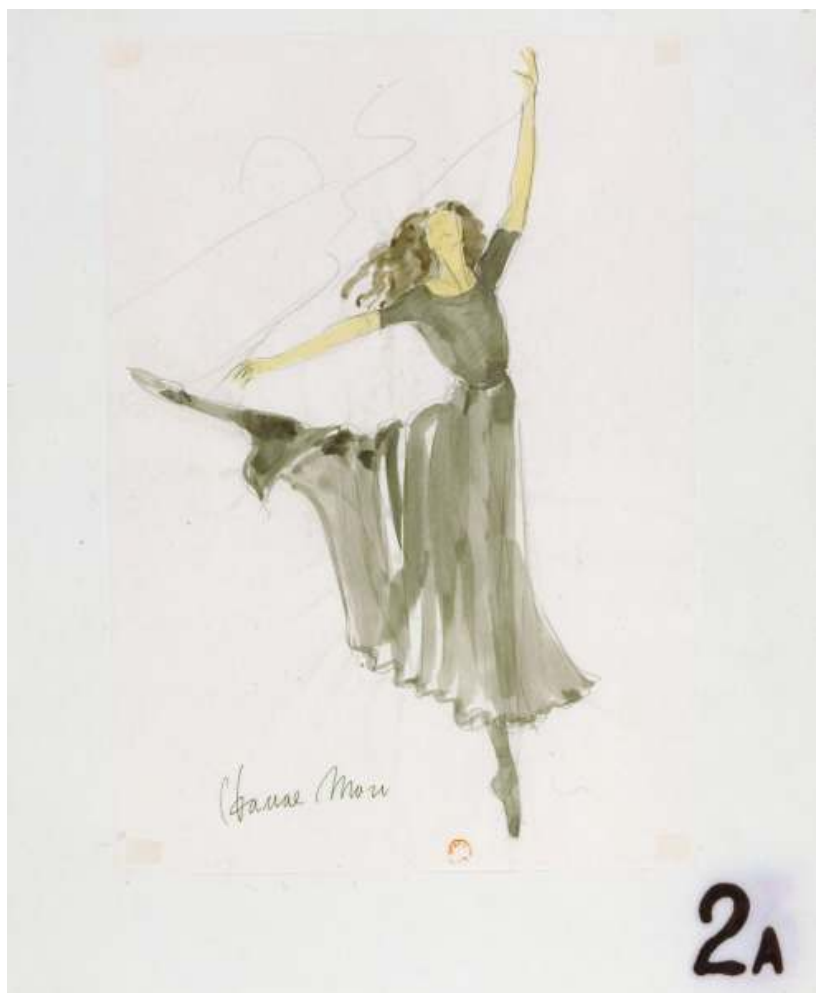

Bibliothèque nationale de France, Bibliothèque-musée de l'Opéra (D216 (156, pl 2A)

(c) Bibliothèque nationale de France

12 Cette création joue de l'hybridation des arts - le cinéma et la danse - pour proposer finalement une mise en abyme du parcours de Noureev lui-même, maltraité par son père et encouragé par une fée - professeur de danse... Cette Cendrillon contemporaine est un hymne à la création, au rêve, et une déclaration d'amour au monde du spectacle qui pouvait lui sembler hors de portée lorsqu'il était encore tout jeune. En associant les arts dans son ballet (musique, danse, cinéma, scénographie et costumes ${ }^{10}$ ), Noureev révèle par l'intermédiaire de son héroïne le pouvoir de la beauté à transfigurer les êtres. 


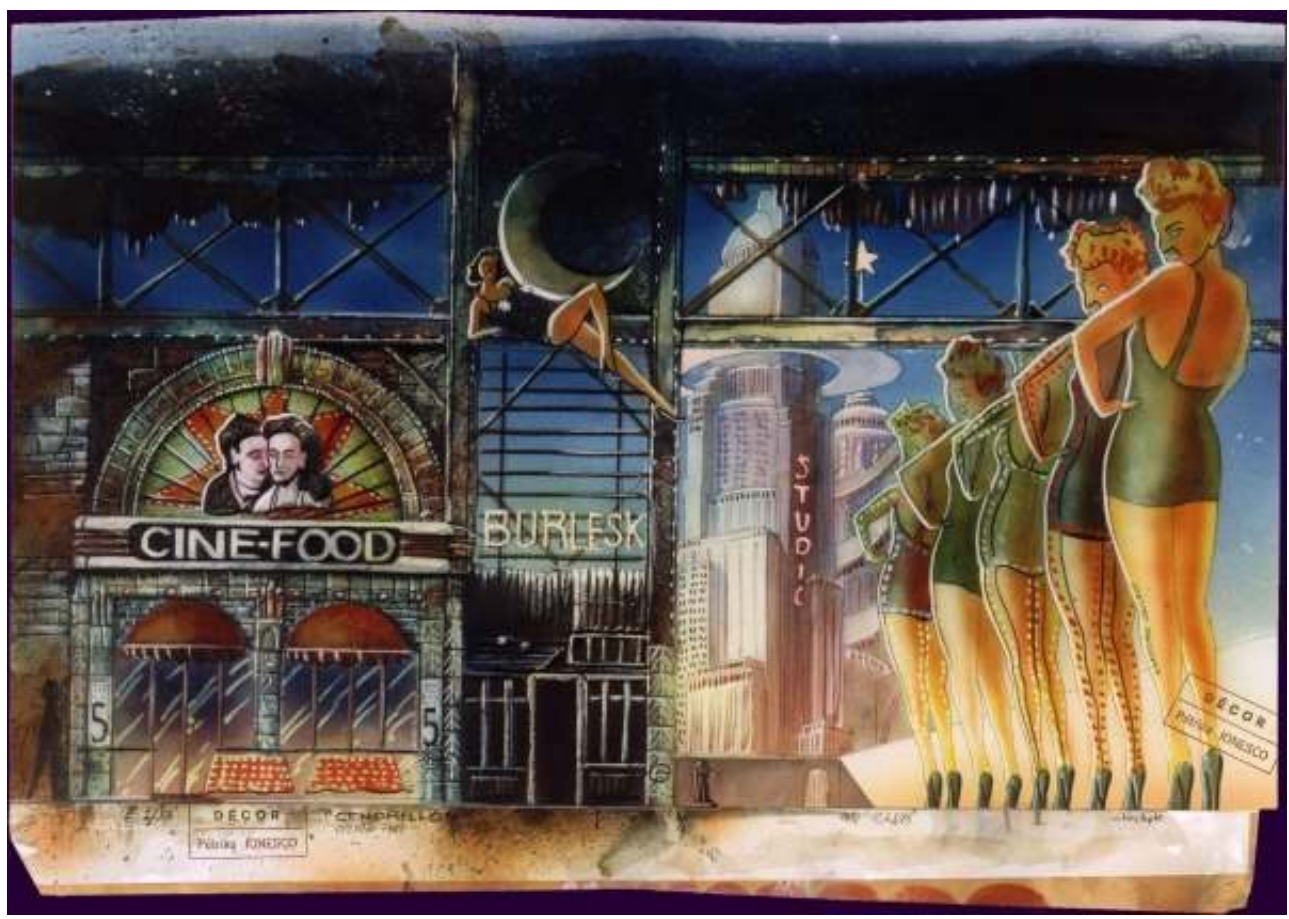

Bibliothèque nationale de France, Bibliothèque-musée de I'Opéra (Esq. 0 1986, Cendrillon, pl. 6)

\section{Cendrillon et l'opéra : une relecture sous le regard de Méliès proposée par Benjamin Lazar}

Lorsqu'il met en scène l'opéra de Massenet à l'Opéra comique en 2011, Benjamin Lazar est particulièrement sensible aux liens qui unissent musique et théâtre; en spécialiste du théâtre baroque ${ }^{11}$, il souligne (par les costumes et le jeu) les références au XVII ${ }^{\mathrm{e}}$ siècle de Massenet et de son librettiste: Perrault, bien sûr, mais aussi Molière et la tragédie lyrique. Cette création lui ouvre cependant un nouvel horizon : la magie des premiers pas du cinématographe. Sa mise en scène se présente d'emblée comme le jeu de miroir d'une époque $\left(\mathrm{XVII}^{\mathrm{e}}\right)$ dans une autre $\left(\mathrm{XIX}^{\mathrm{e}}\right)$ à partir d'un dispositif dramaturgique et scénographique d'emboitement orchestré par un regard contemporain :

Cette distance m'a permis une double ouverture. D'un point de vue géographique tout d'abord, il se trouve que la même année, Georges Méliès présente son film Cendrillon au théâtre Robert Houdin qui se trouve à quelques rues de distance de l'Opéra Comique. C'est plus qu'une coïncidence! C'est pourquoi j'ai imaginé la présence tutélaire de ce créateur. Au niveau de l'imaginaire, le livret contient en germe une série de thèmes et d'obsessions qui parcourent cette fin de siècle : tirer les fils du livret en les mettant en regard de l'époque de sa production permet d'éviter le geste de reconstitution historique pour aller vers ce qu'on pourrait appeler «l'inconscient» de l'œuvre et ouvrir ainsi sur des pistes tout à fait intéressantes. ${ }^{12}$

La fabrique du merveilleux doit beaucoup à l'influence de Méliès ${ }^{13}$ (le carrosse très ouvragé mais aussi la fumée et les effet de flou en noir et blanc) : un merveilleux nimbé de poésie visuelle renvoyant au symbolisme. La force du spectacle repose de fait sur le choc des époques et des arts (musique, chant, théâtre, cinéma) produisant un effet baroque de 
théâtre dans le théâtre, où l'opéra de Massenet devient la matière d'un tournage de la Belle époque sous le regard du spectateur qui ne cesse d'en décrypter les échos. Le modernisme architectural $\mathrm{du} \mathrm{XIX}^{\mathrm{e}}$ siècle est très présent dans la scénographie: la structure métallique rappelle le studio de cinéma de Méliès, la tour Eiffel mais peut aussi suggérer une cage ou une prison... Entre enchantement et inquiétude, les signes sont offerts à l'interprétation. La scène du bal chez le prince est transformée en divertissement et renvoie, quant à elle, au genre de la «revue », par la succession de numéros sans liens entre eux où l'entrée de Cendrillon fait office de « clou » du spectacle.

Cendrillon à l'Opéra Comique, mars 2011

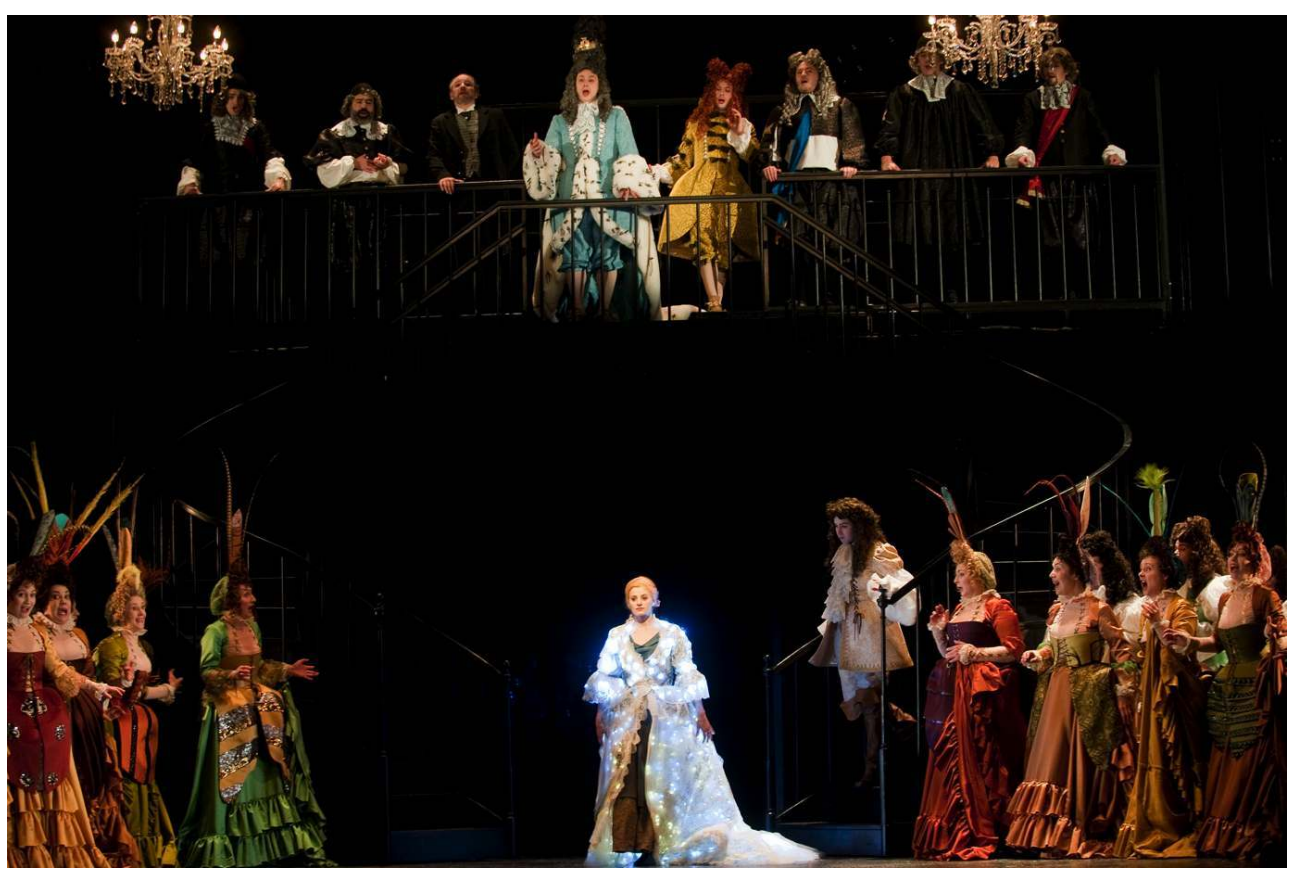

(c) Elizabeth Carecchio

\section{Relectures artistiques des formes classiques: l'irruption de l'inquiétante étrangeté}

\section{Démystifier le merveilleux : L'irruption du réel dans le conte (de fées ?)}

L'intérêt de Freud pour le conte et les travaux de Bruno Bettelheim ${ }^{14}$ ont transformé le regard porté sur le merveilleux qui devient l'émanation d'un désir inconscient masquant le réel. La modernité démystifie le merveilleux qui fait place à l'irruption de l'inquiétante étrangeté dans le réel. La maison de verre dans le spectacle de Joël Pommerat n'est-elle pas une tentative de se protéger du monde ? Une protection inefficace si l'on en juge par le son angoissant de l'impact des oiseaux venant s'écraser sur les parois ${ }^{15}$, tandis que dans le conte de Grimm, les oiseaux étaient un agent du merveilleux. Les relectures contemporaines actualisent ainsi le conte dans des transpositions qui s'attachent moins au merveilleux qu'à ce qui nous est proche et qui nous inquiète. Les frontières ne sont plus aussi nettes entre les bons et les méchants: Cendrillon n'est plus forcément une 
pauvre victime totalement innocente. Ce qui intéresse les artistes est précisément le trouble de ce passage entre un état et un autre. La relecture, en ce qu'elle induit, de fait, une distanciation, permet ce double regard porté sur l'œuvre comme source et sur sa réinterprétation. En s'emparant des œuvres classiques (l'opéra de Massenet ou le ballet de Prokofiev), les artistes contemporains (chorégraphes, metteurs en scènes) mettent en lumière les forces les plus obscures qui se nichent au cœurs de ces œuvres du patrimoine culturel ; l'exhibition de la folie et du grinçant a remplacé le rêve et le merveilleux.

\section{Morbidité et inconscient : l'exhibition spectaculaire}

Le prologue proposé par Benjamin Lazar dans sa mise en scène donne le ton: un homme en redingote arrive devant le rideau rouge et met en marche un phonographe : "Mesdames et messieurs, c'est un mort qui vous parle ", entend-on. Le spectacle se place ainsi sous l'égide de l'invention technologique dans ce qu'elle a de plus étonnant (pour un spectateur du xIX ${ }^{e}$ siècle) mais aussi de charge mortifère, car enregistrer sa voix, c'est bien une manière de se mettre au tombeau vivant. Le spectacle fait ainsi très clairement revenir les morts parmi les vivants. Dans cette atmosphère très « fin de siècle » surgit le fantôme blanc de Cendrillon vêtue d'une longue chemise de nuit. Le traitement du personnage en héroïne du XIX ${ }^{e}$ contraste fortement avec les autres personnages féminins ancrés dans un imaginaire du XVII ${ }^{e}$ siècle, non seulement par le costume, mais aussi par le jeu. La scène où elle s'éveille d'un sommeil lourd et chargé de rêves agités (acte IV, premier tableau, scène 1) renvoie de manière explicite aux séances du docteur Charcot à l'hôpital de la Salpêtrière avec ses patientes : «tu riais... tu pleurais.../Sans motif et sans trêve/Tu vivais comme dans un rêve.../Comme au hasard/Tu murmurais des mots confus... » lui confie son père, ce à quoi la jeune fille répond : "J'étais donc insensée... ». Benjamin Lazar traite la scène de manière clinique: le lit à barreaux fait penser à l'univers médical et l'ambiguïté de la relation avec son père qui la déshabille peut expliquer le basculement vers la folie :

Ses émotions sont exacerbées, elle rit et elle pleure en même temps, elle éprouve des difficultés pour parler et termine par une attaque de nerfs. Ce passage vers l'adolescence marque la mort de l'enfance mais revêt également une dimension fantasmatique. Le spectateur occupe d'une certaine manière une position de voyeur: la jeune femme devient un objet d'observation, un objet clinique. C'est pourquoi j'ai pensé aux expériences que Charcot faisait avec ses malades, notamment avec sa patiente Augustine. Le lit est une reproduction fidèle du modèle utilisé à la Salpêtrière. Les hallucinations acoustiques de Cendrillon viennent peutêtre de ce que la réalité est trop dure pour elle et qu'elle y substitue l'instance du rêve ? ${ }^{16}$ 
Cendrillon à l'Opéra Comique, mars 2011

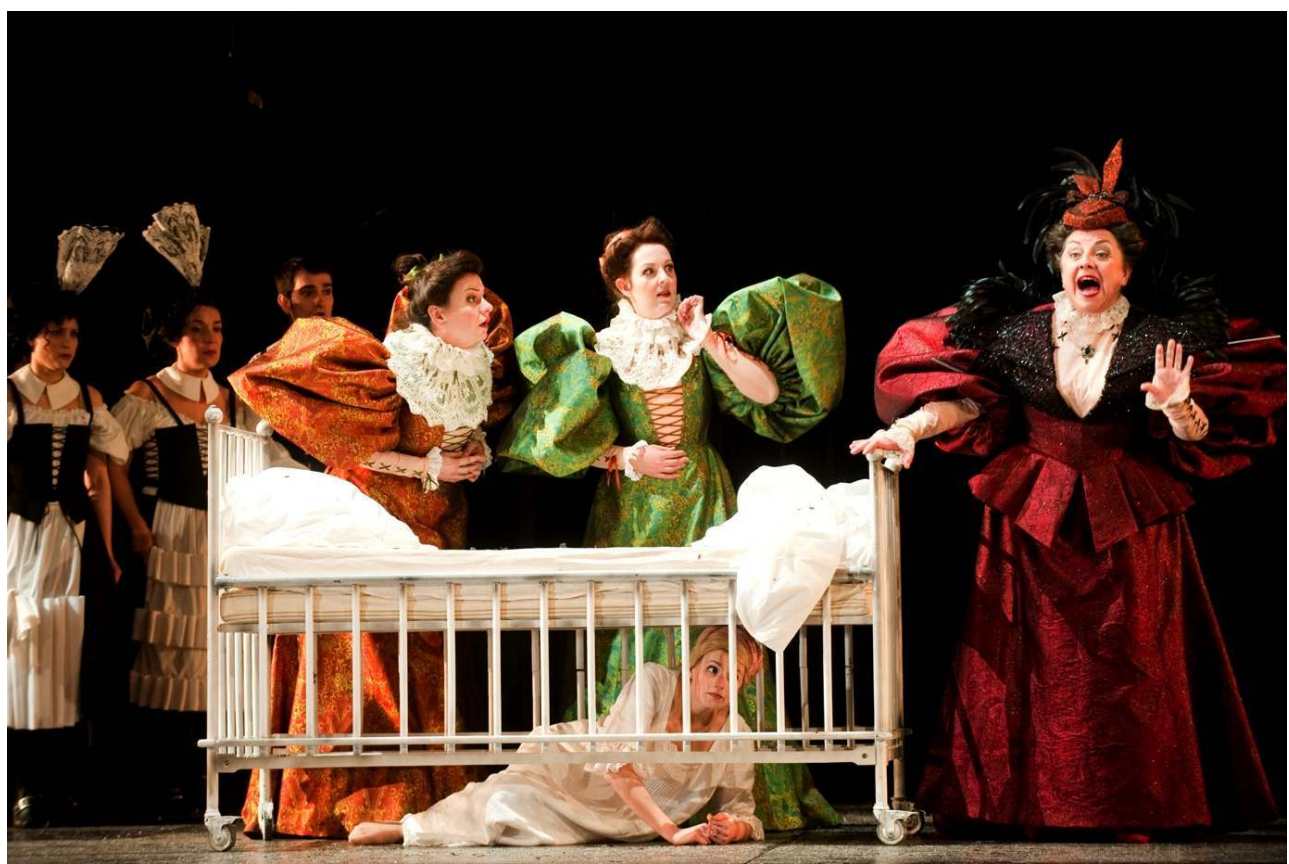

(c) Elizabeth Carecchio

\section{L'enfance et ses cruautés dans le ballet de Maguy Marin}

17 La transposition que Maguy Marin opère dans le ballet de Prokofiev est tout aussi radicale: ce n'est d'ailleurs pas tant l'idée de faire des personnages du conte ceux d'un monde de jouets, idée qui renvoie à l'univers enfantin des ballets comme Casse-Noisette de Tchaïkovski par exemple, mais c'est le traitement lui-même des personnages et la chorégraphie qu'elle écrit pour un corps de ballet des plus classiques - Ballet de l'Opéra de Lyon - qui en fait une version étonnante ${ }^{17}$. Rappelons qu'à sa création, en 1985, ce fut une sorte de révolution dans le monde de la danse et qu'elle rencontra de fortes réticences de la part de danseurs qui appréciaient modérément leurs costumes et cette nouvelle danse délicieusement empotée. Il leur semblait que la danse classique n'était pas respectée, que leur corps de danseurs était niés, et se trouvaient tout simplement grotesques. Tandis que le grinçant faisaient ses premiers pas dans l'institution du ballet classique, la fée Maguy Marin défaisait les fantasmes de jeune étoile. Cette œuvre marque assurément une révolution esthétique dans l'histoire de la danse en ce qu'elle introduit le discordant, et d'une certaine manière la déformation du corps à un endroit où, plus que tout autre, il se doit d'être normé.

18 La scénographie du ballet évoque une grande maison de poupées avec ses compartiments. Nous sommes visiblement dans la chambre d'une petite fille endormie tandis que ses jouets commencent à s'animer. Les danseurs sont camouflés sous un costume rembourré, surmonté d'une tête ronde, rigide, aux grands yeux ouverts sur le monde. Naïfs, maladroits et assez rudimentaires, ces jouets semblent peu soignés par leur propriétaire. Ils n'en sont que mieux réussis par la costumière Montserrat Casanova qui invente un personnage hybride entre la marionnette et le corps dansé. Nue sous son tablier, c'est avec une petite jupette de tulle rose que la poupée Cendrillon ira au bal retrouver le 
Prince. Son apprentissage de la danse la renvoie du côté de l'animalité : tel un jeune poulain qui doit se dresser sur ses longues jambes, elle apprend avec difficulté les pas qui feront d'elle une ballerine remarquée à la cour. La cour comme espace de contraintes devient cour de récréation, lieu de toutes les cruautés enfantines; de nouveau Cendrillon se retrouve à terre, humiliée et rejetée par ses camarades lors d'un banal jeu de corde à sauter qui finit par déraper. Comme on se croit plus aux contes de fées, on ne croit plus non plus à cet état d'innocence de l'enfance. Les jouets entre eux ne sont que le reflet de ce qu'ils subissent de la part des enfants, et la méchante belle-mère porte une profonde entaille sur le visage comme un stigmate de son passé. La maladresse et le burlesque cèdent la place in fine à la grâce et à la beauté, mais le rire s'étrangle dans l'émotion suscitée par une vision si humaine du conte de poupées. En évacuant la virtuosité des figures, et en plaçant au centre de sa dramaturgie la matérialité marionnettique, Maguy Marin recentre le conte vers l'humain, c'est-à-dire vers ce qui est sensible à l'intérieur de chacune de nos carapaces malmenées. N’y aurait-il pas un peu de Grégoire Samsa, le héros de La Métamorphose de Kafka, dans cette Cendrillon revisitée?

Aujourd'hui, si la naïveté peut parfois encore nous pousser à croire au Prince charmant, la fée, elle, a définitivement quitté ses habits du merveilleux. Dans les formes artistiques proposées, davantage que la jeune héroïne, il semble évident que c'est cette figure qui se métamorphose au cours des adaptations (précepteur, mendiant, producteur de cinéma, etc.). Dans le spectacle de Joël Pommerat, la fée cherche coûte que coûte à s'humaniser et à quitter le monde de la magie pour réussir des tours dans le monde réel (celui où il $\mathrm{y} a$ « un truc » qui permet l'illusion). On peut se demander alors, si en s'émancipant du conte, la fée ne prendrait pas la figure du metteur en scène, celui qui cherche à créer de l'illusion avec les matériaux du réel ou celui qui choisit exhiber l'envers de la fabrique théâtrale quitte à placer le spectateur devant sa double contradiction (croire et ne pas croire).

\section{NOTES}

1. Voir dans ce dossier les articles de Marion Boudier et de Marie Bernanoce.

2. L'histoire de Rhodopis, contée par Strabon et retranscrite par Elien au III ${ }^{\mathrm{e}}$ siècle selon Adolphe ADERER, «Cendrillon au théâtre », in Le Théâtre, 19 juillet 1899, p 8.

3. Ce genre peu abordé jusqu'à présent est l'objet d'une étude menée par Roxane Martin : La Féérie romantique sur les scène parisiennes (1791-1864), Paris, Champion, 2007.

4. Cité dans le dossier Cendrillon, collection « Opéra en acte », SCÉRÉN [CNDP-CRDP], 2011, URL : http://www.reseau-canope.fr/opera-en-actes/les-operas/cendrillon/une-cendrillon-fin-desiecle/une-oeuvre-emblematique-de-la-belle-epoque/

5. Le Ménestrel, mai 1899, cité dans le dossier Cendrillon, ibid.

6. Agnès Terrier, Cendrillon, Programme de l'Opéra Comique, janvier 2011.

7. Noémie Courtès les énumère assez précisément dans son article «Cendrillon mise en pièces ou la seconde immortalité de Perrault au XIX ${ }^{\mathrm{e}}$ siècle », Féeries, 4/2007, p. 73-88.

8. Avant, il y a eu Marius Petipa (1893) et Mikhail Fokine avec sa Cinderella interprétée par le Ballet Russe et présentée à Londres en 1938. 
9. Rudolf Noureev - 1986, site de la fondation, URL : http://www.noureev.org

10. Les costumes sont de Hanae Mori.

11. Voir sa mise en scène du Bourgeois Gentilhomme, comédie-ballet de Molière et de Lully, DVD Alpha /Arte, 2005.

12. Cendrillon, Programme de l'Opéra Comique, janvier 2011.

13. On peut voir le film de Méliès sur internet, URL: https://www.youtube.com/watch ? $\mathrm{v}=5 \mathrm{Sb} 3 q$ piMcwE

14. Bruno Bettelheim, Psychanalyse des contes de fées, Hachette Littérature, collection "Pluriel ", 1998.

15. Ces oiseaux qui étaient chez Grimm les agents du merveilleux.

16. Propos recueillis par Rafaëlle Jolivet Pignon, dossier Cendrillon, collection « Opéra en acte »,. op. cit.

17. Cendrillon, ballet de Maguy Marin, DVD, La Sept/Channel 4/ ZDF/Studio Production de l'Opéra de Lyon, 1989. 